on the environmental serum concentrations. Careful quantification of the response rates for growth factors suggested that under an appropriate environmental condition to bring optimal heterogeneity caused by the stochasticity of cell fate decisions, the effects of the growth factors become maximum, i.e., suppressing cell death, NGF directs PC12 cell fates efficiently to differentiation and EGF directs to proliferation.

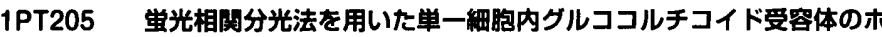 モダイマー形成の解析}

Quantification of Glucocorticoid Receptor of homo-dimer in single cell by using Fluorescence Correlation Spectroscopy

Sho Oasa ${ }^{1}$, Akira Sasaki ${ }^{1}$, Shintaro Mikuni ${ }^{1,2}$, Masataka Kinjo ${ }^{1}\left({ }^{1}\right.$ Grad. Life Sci., Hokkaido Univ., ${ }^{2}$ Grad. Med. Department of Advanced Optical Imaging Reasarch., Hokkaido Univ.)

The dimerization of GR (Glucocorticoid Receptor) in single cell is analysed. It is known that GR is dimerized by adding Dex (Dexamethasone) in living cell. However, when and where the dimerization of GR is occurred is still controversial. Moreover, many previous studies of GR processing have been carried out by using trangent-expressed cell. When the heterogeneous amounts of target protein is estimated in each cell such as trangent-expressed cell, the single-cell measurement might be better than conventional biochemical methods and required for better understanding of GR activity. In our laboratory, to assess protein contents in single cell, the small cavity (MicroWell) on the PDMS (polydimethylsiloxane) chip was constructed $[1,2]$. Single cell was easily isolated in MicroWell and target protein was extracted and kept into the MicroWell. FCS (Fluorescence Correlation Spectroscopy) was performed to obtain number and concentration of target protein in MicroWell and protein complex information from CPM (count per molecule). EGFP-GR dimerized by adding Dex is analysed by using CPM and the ratio between monomeric and dimeric EGFP-GR is estimated with different concentraton of EGFP-GR in trangent-expressed cell.

[1] Sasaki, A, Kinjo, M, Curr Pharm Biotechnol., 2010, 11, 117-121

[2] Oasa, S, Sasaki, A, Kinjo, M, Biophysic Society meething, 2011

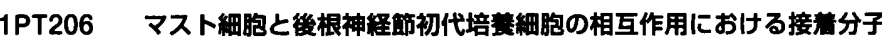 の研究}

Cell adhesion molecule 1 (CADM1) on mast cells promotes interaction with dorsal root ganglion neurites by heterophilic binding to nectin-3

Tadahide, Furuno ${ }^{1}$, Miho, Sekimura ${ }^{2}$, Keisuke, Okamoto $^{2}$, Man, Hagiyama ${ }^{3}$, Akihiko, Ito ${ }^{3}$, Ryo, Suzuki ${ }^{2}$, Naohide, Hirashima ${ }^{2}$, Mamoru, Nakanishi ${ }^{1}{ }^{1} S c h$ Pharm., Aichi Gakuin Univ., ${ }^{2}$ Grad. Sch. Pharm. Sci., Nagoya City Univ., ${ }^{3}$ Fac. Med., Kinki Univ.)

Functional interaction between mast cells and sensory nerves has been shown to occur in various physiological and pathological situations. We previously showed that an immunoglobulin superfamily adhesion molecule, cell adhesion molecule 1 (CADM1), on both mast cells and sympathetic superior cervical ganglia (SCG) promoted their attachment and communication via the homophilic binding using in vitro coculture approach. Here we have studied the role of CADM1 in the interaction between sensory dorsal root ganglia (DRG) and mast cells. Though CADMl was hardly expressed in DRG, CADMI on bone marrow-derived mast cells (BMMCs) accumulated in contact region with DRG neurites. CADM1-deficient IC-2 mast cell line was defective in attachment to DRG, while it was recovered in CADM1-transfected IC-2 cells (IC-2 $2^{\mathrm{CADM} 1}$ cells) to the similar level to BMMCs. Searching adhesion molecules known as heterophilic binding partner of CADM1, DRG expressed nectin-3 but not Necl-1, Necl-5, and class-I-restricted T-cell-associated molecule. CADM1 on mast cells and nectin-3 on DRG neurites were colocalized in their attached region in coculture. The efficiency in calcium response of attached IC-2CADM1 cells after specific activation of DRG was significantly higher than that of IC-2 cells and was as similar as BMMCs. A neutralizing antibody to nectin-3 inhibited both mast cell attachment and subsequent calcium responses. This suggests that heterophilic binding between CADM1 and nectin-3 mediates functional DRG-mast cell interactions in vitro.

1PT207 Duration of CaMKII activation required for plasticity of dendritic spines revealed by photo-activatable CaMKII inhibitor
Hideji Murakoshi ${ }^{1,2}$, Ryohei Yasuda ${ }^{3}\left(^{1}\right.$ National Institute for Physiological Sciences, ${ }^{2}$ PRESTO, JST, ${ }^{3}$ Dept of Neurobiology, Howard Hughes Medical Institute, Duke University Medical Center)

$\mathrm{Ca} 2+/$ Calmodulin-dependent kinase II (CaMKII) is required for long-term potentiation (LTP) and associated spine enlargement, which underlies learning and memory in the mammalian forebrain. Previously, it has been demonstrated that exogenous CaMKII activity lasts for $\sim 1$ min during induction of spinespecific LTP and spine enlargement. Here, to determine the duration of endogenous CaMKII activation required for CaMKII-dependent synaptic plasticity, we developed a genetically encoded light-inducible inhibitor of CaMKII activation by fusing autocamtide inhibitory peptide 2 (AIP2) to LOV2$\mathrm{J} \alpha$ derived from phototropin $\mathrm{l}$. We applied it to the study of structural plasticity of single dendritic spines by using 2-photon glutamate uncaging, and found that $\sim 10 \mathrm{~s}$ of CaMKII activation is sufficient for inducing transient spine enlargement, while $\sim 60 \mathrm{~s}$ is required for sustained spine enlargement. The different temporal requirement between these two phases must be due to differences in the integration properties of downstream signaling. The design of the light-inducible CaMKII inhibitor shown here should be applicable to the design of similar light-inducible inhibitors for many different proteins.

\section{PT208 TPO 受容体の二目体安定化によるシグナルの正のフィードバック} 制御

Positive feedback control of thrombopoietin signaling by stabilization of its receptor dimers revealed through singlemolecule imaging

Akihiko Sakamoto ${ }^{1}$, Takashi Kato ${ }^{2}$, Takashi Funatsu ${ }^{1}\left({ }^{1}\right.$ Grad. Sch. of Pharm. Sci., The Univ. of Tokyo, ${ }^{2}$ Fac. of Ed. and Int. Arts. and Sci., Waseda Univ.)

The thrombopoietin (TPO) receptor, $\mathrm{Mpl}$, is a single transmembrane cytokine receptor which stimulates differentiation of platelet progenitor cells and maintains hematopoietic stem cells. Like other cytokines, TPO induces phosphorylation of $\mathrm{Mpl}$, which recruits various adaptor proteins regulating cell proliferation and differentiation. Although dimerization of cytokine receptors is necessary for their phosphorylation, the regulatory mechanism is poorly understood. Here, we describe the regulatory mechanism of Mpl dimerization by single-molecule fluorescence imaging in living cells. Fluorescently labeled $\mathrm{Mpl}$ molecules associated and dissociated repeatedly on the plasma membrane. As a result, $\mathrm{Mpl}$ molecules resided in monomers, dimers, and oligomers. TPO increased the ratio of $\mathrm{Mpl}$ dimers or oligomers, which was resulted from stabilization of $\mathrm{Mpl}$ dimers. Surprisingly, stabilization of Mpl dimers was resulted from their phosphorylation rather than TPO binding itself. Furthermore, TPO-induced stabilization of $\mathrm{Mpl}$ dimers was dependent on an adaptor protein, Shc, suggesting that phosphorylated Mpl dimers were crosslinked by Shc. By contrast, a monomeric Mpl mutant inhibited TPO-induced phosphorylation of $\mathrm{Mpl}$ and downstream cell proliferation. In addition, cholesterol depletion reduced the stability of $\mathrm{Mpl}$ dimers, resulting in inhibition of their phosphorylation. Thus, we propose positive feedback control of TPO signaling by stabilization of $\mathrm{Mpl}$ dimers.

\section{PT209 Rac1 recruitment to the archipelago structure of focal} adhesion through the fluid membrane as revealed by singlemolecule analysis

Akihiro Shibata ${ }^{1}$, Chen Limin ${ }^{1}$, Nagai Rie ${ }^{1}$, Fumiyoshi Ishidate', Yoshihiro $\mathrm{Miwa}^{2}$, Keiji Naruse ${ }^{3}$, Takahiro Fujiwara ${ }^{1}$, Akihiro Kusumi ${ }^{1}$ ('Institute for Frontier Medical Sciences, Kyoto University, ${ }^{2}$ Department of Pharmacology, Institute of Basic Medical Sciences, University of Tsukuba, ${ }^{3}$ Cardiovascular Physiology, Okayama University Graduate School of Medicine, Dentistry, and Pharmaceutical Sciences)

The small G protein Racl has been shown to regulate actin reorganization and polymerization at the focal adhesions (FA), an integrin-based architecture of the plasma membrane (PM), mechanically linking the extracellular matrix with the termini of actin stress fibers, providing the scaffold for cell migration. Previously, we found that the FA is not a single entity of massive protein assembly, but rather consists of many protein islands sparsely scattered in the fluid PM (archipelago architecture), and membrane molecules undergo rapid diffusion in the PM even within the FA zone. Here, using single fluorescent-molecule imaging, we examined how Racl and its upstream activator GEFs, alphaPIX and betaPIX, are recruited to the FA. We found that almost all of the PIX molecules were directly recruited to the FA zone from the cytoplasm. Meanwhile, $81 \%$ of Rac1, which has a geranylgeranyl (+ possibly a palmitoyl) hydrophobic anchoring chains, first landed on the general inner surface of the PM from the cytoplasm and then 